\title{
Electroweak production of Higgs boson pairs in 2HDMs
}

\section{Rikard Enberg}

Department of Physics and Astronomy, Uppsala University, Box 516, SE-751 20 Uppsala, Sweden

E-mail: rikard.enberg@physics.uu.se

\section{William Klemm}

School of Physics \& Astronomy, University of Manchester, Manchester M13 9PL, UK

E-mail: william.klemmephysics.uu.se

\section{Stefano Moretti}

School of Physics \& Astronomy, University of Southampton, Southampton SO17 1BJ, UK

E-mail: s.morettiesoton.ac.uk

\section{Shoaib Munir*}

School of Physics, Korea Institute for Advanced Study, Seoul 130-722, Republic of Korea

E-mail: smunirakias.re.kr

\begin{abstract}
One of the main features of a Two-Higgs Doublet Model (2HDM) is the presence of two additional neutral Higgs states, besides the one mimicking the $\sim 125 \mathrm{GeV}$ state observed at the LHC. The three Higgs bosons of a $2 \mathrm{HDM}$ can be produced at the LHC either singly via gluon fusion or in pairs with each other. When analyzing their pair production, the emphasis is laid on gluoninitiated processes, and the electroweak (EW) production is generally not treated on the same footing, assuming its contribution to be highly subleading. We show here that when the sum of the masses of the lightest scalar and pseudoscalar Higgs bosons in the Type-I 2HDM is smaller than the Z-boson mass, their EW pair production can dominate over QCD pair production by orders of magnitude.
\end{abstract}

38th International Conference on High Energy Physics

3-10 August 2016

Chicago, USA

${ }^{*}$ Speaker. 


\section{Light Higgs bosons in the Type-I 2HDM}

In the Type-I 2HDM only one of the two Higgs doublets, $\phi_{1}$ and $\phi_{2}$, couples to all the Standard Model (SM) fermions, with a $Z_{2}$ symmetry preventing large flavor changing neutral currents. The model contains three neutral Higgs states, two scalars, $h$ and $H$, with $m_{h}<m_{H}$, and a pseudoscalar, A. Either one of $h$ or $H$ can play the role of the SM-like Higgs boson, $h_{\mathrm{obs}}$, discovered at the LHC $[1,2]$. In the scenario when the mass and signal rates of $H$ are consistent with those of $h_{\mathrm{obs}}, h$ can be as light as a few $\mathrm{GeV}$, without violating the constraints from negative searches at the LEP collider, Tevatron and LHC. When the $A$ is additionally light enough that $m_{h}+m_{A}<m_{Z}$, their pairproduction via a resonant $Z$ in the $s$-channel becomes possible, but only in the $q \bar{q}$-fusion process, since it is prohibited in the gluon-fusion process by the Landau-Yang theorem [3, 4]. As a result, the production cross section of the $h A$ pair gets considerably enhanced below the $Z$ mass.

\section{Numerical analysis}

To analyse the significance of the EW $h A$ pair-production, we first performed a numerical scan of the six free parameters of the Type-I 2HDM using the 2HDMC-v1.7.0 [5] program, in order to find points with $m_{h}+m_{A}<m_{Z}$ that are consistent with the results from collider searches as well as from $b$-physics and EW precision experiments. These parameters include $m_{h}, m_{A}, m_{H^{ \pm}}, \sin (\beta-$ $\alpha), m_{12}^{2}$ and $\tan \beta$, with $m_{H}$ fixed to $125 \mathrm{GeV}$. A complete list of the paramater ranges and the

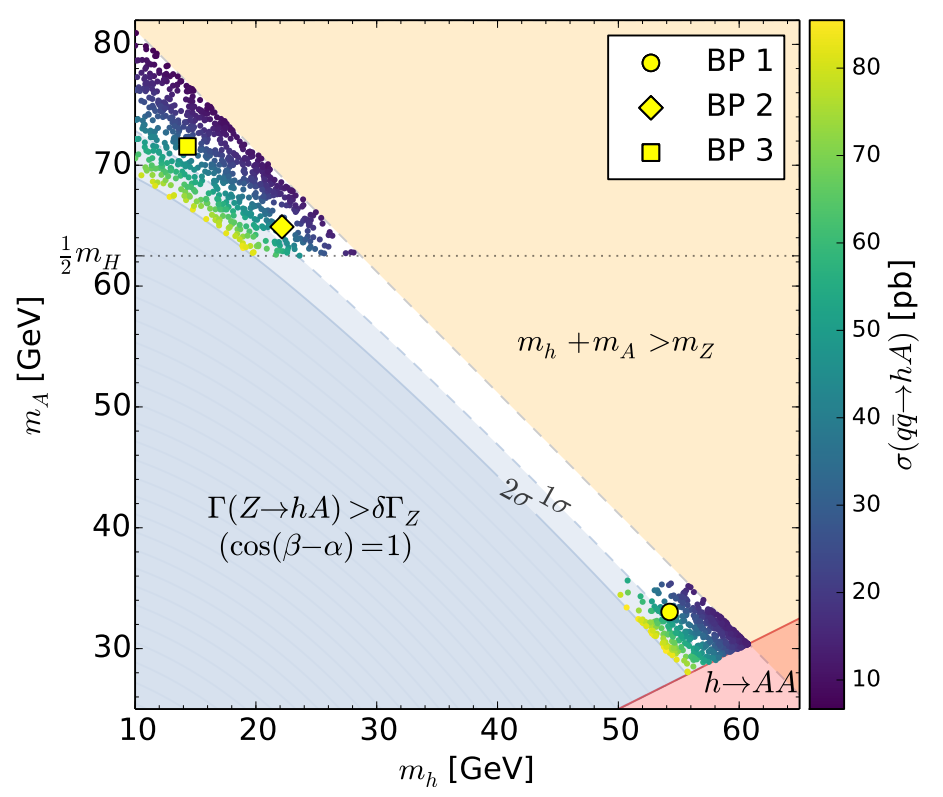

Figure 1: Points satisfying all the constraints imposed during the scan and additionally lying within the experimental uncertainty on the $Z \rightarrow h A$ partial width, at the $1 \sigma$ (lighter) and $2 \sigma$ (darker) levels, assuming $\cos (\beta-\alpha)=1$. The small red region corresponds to $m_{h}>2 m_{A}$, allowing $h \rightarrow A A$ decays. The three benchmark points have been highlighted in yellow, and the color map corresponds to the total cross section. for the $q \bar{q} \rightarrow h A$ process at $\sqrt{s}=13 \mathrm{TeV}$. 

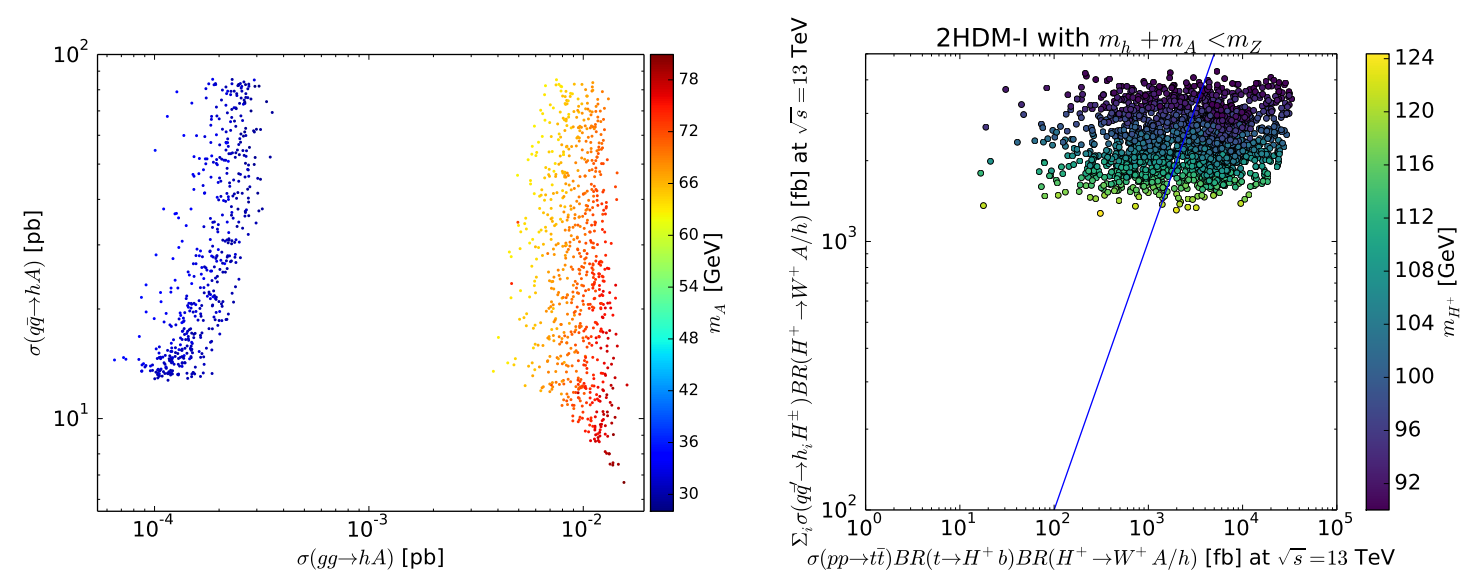

Figure 2: Left: Cross sections for QCD vs. EW production of $h A$ pairs at the LHC, for the good points from the parameter space scan of Type-I 2HDM, with the color map showing the mass of $A$. Right: Cross sections for two of the main production modes of the accompanying $H^{ \pm}$, which decays via $W^{ \pm} h$ or $W^{ \pm} A$ and its mass is indicated by the color map.

constraints imposed in the scan can be found in [6]. In Fig. 1 we show the points passing all these constraints and, additionally, lying within the $2 \sigma$ error on the experimental measurement of the $Z$ width. The ones highlighted in yellow are the benchmark points (BPs) selected for a more detailed investigation. The color map shows the production cross section for the $q \bar{q} \rightarrow h A$ process, calculated using [7].

Fig. 2(left) shows that the production cross section for the at the LHC with $\sqrt{s}=13 \mathrm{TeV}$ can exceed that for the $g g \rightarrow h A$ one, calculated using [8], by a few orders of magnitude, reaching up to about $90 \mathrm{pb}$. Table 1 shows the cross sections corresponding to the two production modes for the three BPs noted earlier. For BP1, where $m_{A}<m_{h}$, the difference between the cross sections is much more enhanced compared to that for the other two BPs, which correspond ot the case $m_{h}<m_{A}$. The table also contains the branching ratios (BRs) of the $h$ and $A$ thus produced in their two most dominant decay modes. Clearly, when kinematically allowed, $Z^{*} A$ is the primary decay channel of $h$ (for BP1) and $Z^{*} h$ of $A$ (for BP2 and BP3). Thus, a non-conventional final state like $Z^{*} b \bar{b} b \bar{b}$ could serve as an important probe of this model scenario.

Finally, a crucial feature of such light $h$ and $A$ is that, in order to satisfy the EW precision constraints, they are always accompanied by a light $H^{ \pm}$. The latter decays dominantly in the $W^{ \pm} h$ or $W^{ \pm} A$ channels, with their combined BR approaching unity. The most significant production process(es) of $H^{ \pm}$, which subsequently decays in one of these two channels, can therefore have a substantial cross section at the LHC [9], as shown in Fig. 2(right). It can thus potentially provide a complimentary signature of the Type-I 2HDM scenario considered here.

\section{References}

[1] G. Aad et al. [ATLAS Collaboration], Observation of a new particle in the search for the Standard Model Higgs boson with the ATLAS detector at the LHC, Phys. Lett. B 716 (2012) 1 [1207. 7214 [hep-ex]]. 


\begin{tabular}{cccccccc}
\hline $\mathrm{BP}$ & $m_{h}$ & $m_{A}$ & $m_{H_{ \pm}}$ & $\sigma(q \bar{q})$ & $\sigma(g g)$ & $\mathrm{BR}\left(h \rightarrow Z^{*} A, b \bar{b}\right)$ & $\mathrm{BR}\left(A \rightarrow Z^{*} h, b \bar{b}\right)$ \\
\hline 1 & 54.2 & 33.0 & 118.3 & 41.2 & $1.5 \times 10^{-4}$ & $0.94,0.05$ & $0,0.86$ \\
2 & 22.2 & 64.9 & 101.5 & 34.4 & $7.2 \times 10^{-3}$ & $0,0.83$ & $0.86,0.12$ \\
3 & 14.3 & 71.6 & 107.2 & 31.6 & $1.1 \times 10^{-2}$ & $0,0.60$ & $0.90,0.08$ \\
\hline
\end{tabular}

Table 1: Parton-level production cross sections (in pb) of $h$ and $A$ pairs, and their largest branching ratios, corresponding to the three selected benchmark points.

[2] S. Chatrchyan et al. [CMS Collaboration], Observation of a new boson at a mass of $125 \mathrm{GeV}$ with the CMS experiment at the LHC, Phys. Lett. B 716 (2012) 30 [1207. 7235 [hep-ex] ].

[3] L. D. Landau, On the angular momentum of a system of two photons, Dokl. Akad. Nauk Ser. Fiz. 60 no. 2 (1948) 207.

[4] C. N. Yang, Selection Rules for the Dematerialization of a Particle Into Two Photons, Phys. Rev. 77 (1950) 242.

[5] D. Eriksson, J. Rathsman and O. Stål, 2HDMC: Two-Higgs-Doublet Model Calculator Physics and Manual, Comput. Phys. Commun. 181 (2010) 189 [0902.0851 [hep-ph] ].

[6] R. Enberg, W. Klemm, S. Moretti and S. Munir, Electroweak production of light scalar-pseudoscalar pairs from extended Higgs sectors, arXiv:1605.02498 [hep-ph] .

[7] J. Alwall et al., The automated computation of tree-level and next-to-leading order differential cross sections, and their matching to parton shower simulations, JHEP 1407 (2014) 079 [1405. 0301 [hep-ph]].

[8] B. Hespel, D. Lopez-Val and E. Vryonidou, Higgs pair production via gluon fusion in the Two-Higgs-Doublet Model, JHEP 1409 (2014) 124 [1407.0281 [hep-ph] ].

[9] A. Arhrib, R. Benbrik, R. Enberg, W. Klemm, S. Moretti and S. Munir, A light charged Higgs boson in the Type-I $2 H D M$, in preparation. 